\title{
New Insights in High-Energy Electron Emission and Underlying Transport Physics of Nanocrystalline Si
}

\author{
Shigeyasu Uno, Member, IEEE, Kazuo Nakazato, Member, IEEE, Shinya Yamaguchi, Akira Kojima, \\ Nobuyoshi Koshida, and Hiroshi Mizuta, Member, IEEE
}

\begin{abstract}
This paper presents quantitative analysis of electron emission from nanocrystalline Si dots, and discusses its mechanism based on the calculations of electronic and phononic states. Analysis of emission energy distribution measured from the vacuum level shows that the energy at the peak of the distribution increases linearly with increasing voltage applied across the nanocrystalline Si system. The slope of the linear law is unity, regardless of process conditions. Increasing voltage significantly changes the shape of the distribution at the energies smaller than the peak, while it has minimal impact at the energies larger than the peak. Both the conventional field emission model and the metal-oxide-semiconductor model fail to explain those behaviors. Calculations of electronic and phononic states in a chain of the nanocrystalline $\mathrm{Si}$ dots indicate a possibility of strong suppression of electron energy relaxation, which may be a possible mechanism of the high-energy electron emission phenomena.
\end{abstract}

Index Terms-Electron emission, nanocrystal, porous silicon, phonons, electrons.

\section{INTRODUCTION}

$\mathbf{I}$ T IS WIDELY known that high-porosity porous silicon (PS) comprises tree-like network of many nanometer size silicon quantum dots surrounded by thin oxide layer of a few nm (silicon nanocrystallites, nanocrystalline silicon: nc-Si). Recently, high-energy electron emission from the PS diode was reported [1], which is schematically illustrated in Fig. 1(a). The silicon substrate is electrochemically treated to form $1 \mu \mathrm{m}$ thick porous silicon region. On top of the surface, 5-nm-thick Au electrode is formed. A positive bias applied to the Au electrode ejects electrons from the emitting surface into the vacuum. The emission energy distribution measured from the vacuum level exhibits a peak, which shifts toward higher energy with increasing positive bias [2], [3] as illustrated in Fig. 1(b). The PS diode is a potential candidate for cold cathodes in future flat panel displays due to its low turn-on voltage and high current stability. Therefore, the electron emission from the PS diodes has been intensively

Manuscript received September 4, 2003; revised September 7, 2003. This paper was presented in part at the IEEE Silicon Nanoelectronics Workshop, Kyoto, Japan, June 2003.

S. Uno, K. Nakazato, and H. Mizuta are with Hitachi Cambridge Laboratory, Hitachi Europe Ltd., Cambridge CB3 OHE, U.K., and also with CREST JST, Tokyo, Japan (e-mail: drsuno@phy.cam.ac.uk).

S. Yamaguchi is with Hitachi Central Research Laboratory, Hitachi Ltd., Tokyo 185-8601, Japan, and also with CREST JST, Tokyo, Japan.

A. Kojima is with Quantum14, Tokyo 184, Japan, and is also with CREST JST, Tokyo, Japan.

N. Koshida is with Division of Electronic and Information Engineering, Faculty of Technology, Tokyo University of Agriculture and Technology, Tokyo 184, Japan, and also with CREST JST, Tokyo, Japan.

Digital Object Identifier 10.1109/TNANO.2003.820508

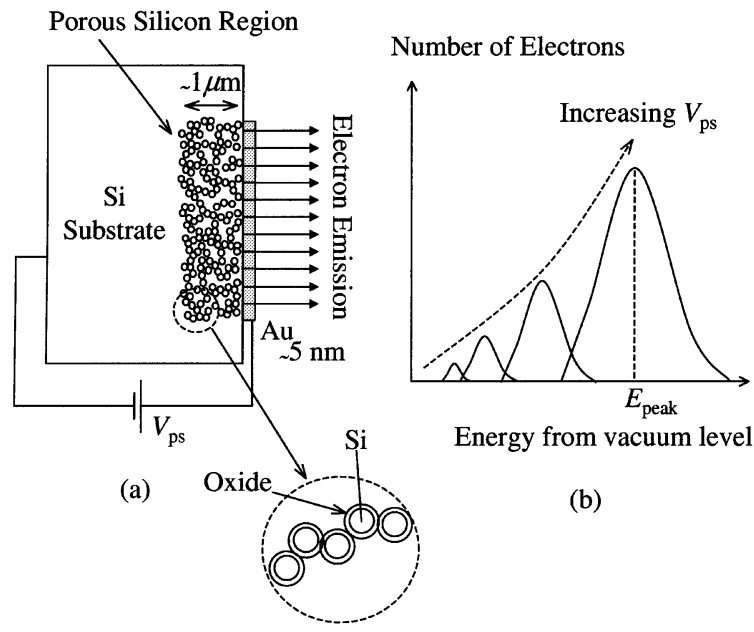

Fig. 1. (a) Schematic Illustration of the electron emission from porous silicon diode under positive bias, $V_{\mathrm{ps}}$. (b) Energy distributions of the emitted electrons measured from the vacuum level under various applied voltage. The energy at the peak of the distribution is denoted as $E_{\text {peak }}$.

investigated for the last several years. However, there are certain aspects that had yet to be satisfactorily examined, including the dependence of the emission energy and its distribution on the voltage applied to the PS diodes. Furthermore, previously suggested models [2], [4] have not been satisfactorily assessed yet.

In this paper, we analyze the relationship between the peak energy and the voltage applied to the PS diode, as well as the dependence of the distribution shape on the applied voltage. Several models are discussed based on the analysis. Finally, we calculate electron wave functions and phonon normal modes in a chain of the nc-Si dots to examine a microscopic mechanism of the high-energy electron emission phenomena.

\section{ANALYSIS OF THE EMISSION ENERGY DISTRIBUTION}

Fig. 2 shows a conduction band profile of the PS diode under positive bias $V_{\text {ps }}(10-20 \mathrm{~V})$ applied to the Au electrode. Major voltage drop occurs across the PS region due to its high resistivity [1], and therefore, Fermi energy difference across the PS region is given by $e V_{\mathrm{ps}}$. Electrons in the silicon substrate are injected into the PS region, and travel toward the emitting surface. Some of those electrons have enough energy to overcome the work function of $\mathrm{Au}, W_{\mathrm{Au}}(\sim 5 \mathrm{eV})$, and are emitted into the vacuum with certain energy distribution. The peak energy measured from the vacuum level, $E_{\text {peak }}$, can be written as,

$$
E_{\text {peak }}=e V_{\mathrm{ps}}-W_{\mathrm{Au}}-E_{\mathrm{loss}},
$$




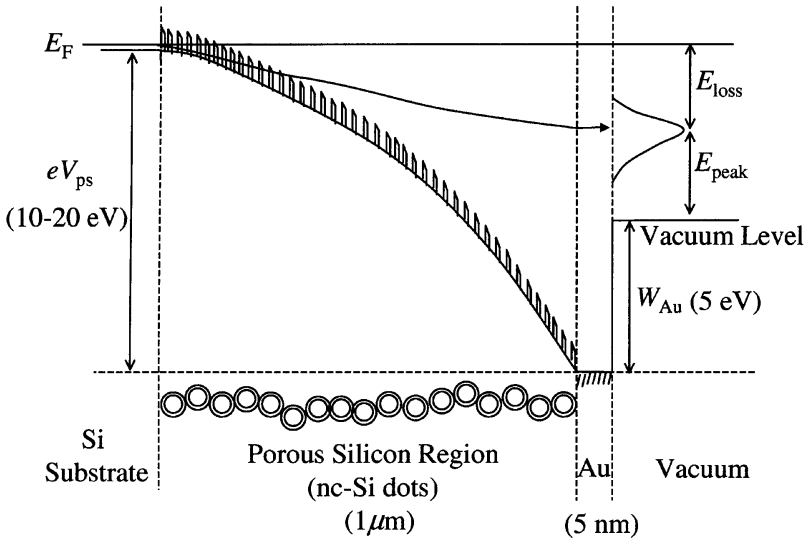

Fig. 2. A conduction band profile under positive bias $V_{\mathrm{ps}}$. $W_{\mathrm{Au}}$ and $E_{\mathrm{loss}}$ denote the work function of Au and the energy difference between the Fermi level at the silicon substrate, and the peak energy level, respectively.

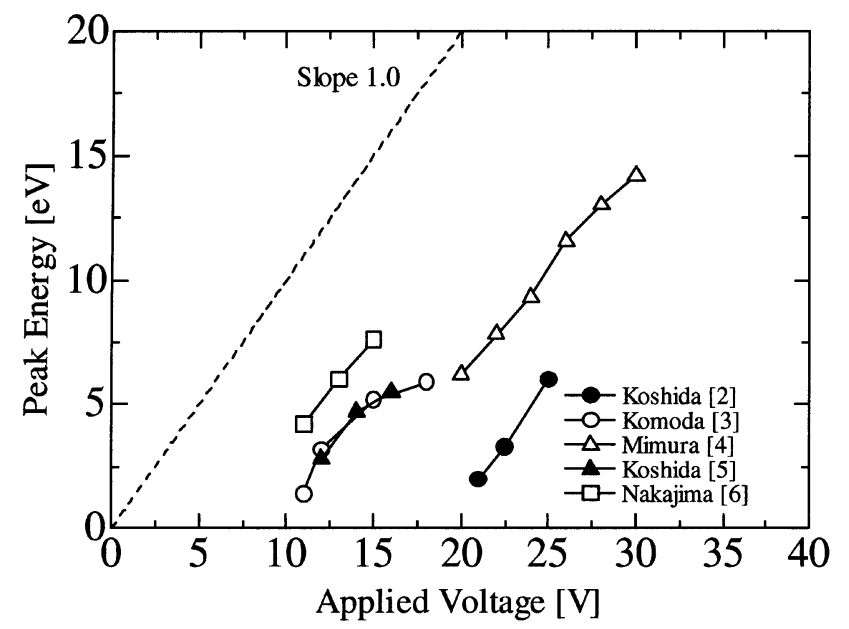

Fig. 3. The peak energy $E_{\text {peak }}$ plotted as a function of applied voltage, showing linear law with unity slope.

where $E_{\text {loss }}$ denotes the energy difference between the Fermi level at the substrate and the peak energy.

Fig. 3 shows the peak energy $E_{\text {peak }}$ as a function of the applied voltage $V_{\mathrm{ps}}$ plotted for various PS diodes, showing virtually linear dependence of $E_{\text {peak }}$ on $V_{\mathrm{ps}}$. The data were taken from the literatures [2]-[6]. It is important to note that the slope is unity for all the PS diodes, irrespective of device structure or fabrication process. In general, $E_{\text {loss }}$ in (1) is a function of $V_{\mathrm{ps}}$ and depends on nature of electron transport in the PS region. The linear law with unity slope, however, indicates that $E_{\text {loss }}$ is nearly independent of $V_{\mathrm{ps}}$, and is a virtually constant parameter of each individual device. This is confirmed in Fig. 4, where the $E_{\text {loss }}$, calculated from the data in Fig. 3 using (1), is nearly independent of $V_{\mathrm{ps}}$.

Fig. 5 shows the impact of $V_{\mathrm{ps}}$ on the energy distribution shape of a PS diode. The horizontal axis is the energy measured from $E_{\text {peak }}$, and the vertical axis is normalized so that the number of electrons at the peak equals unity. The energy dispersion varies depending on $V_{\mathrm{ps}}$. Note that the increasing $V_{\mathrm{ps}}$ dramatically increases the normalized number of electrons at the energies smaller than $E_{\text {peak }}$, while it has minimal impact at the energies larger than $E_{\text {peak }}$. This behavior is quite different

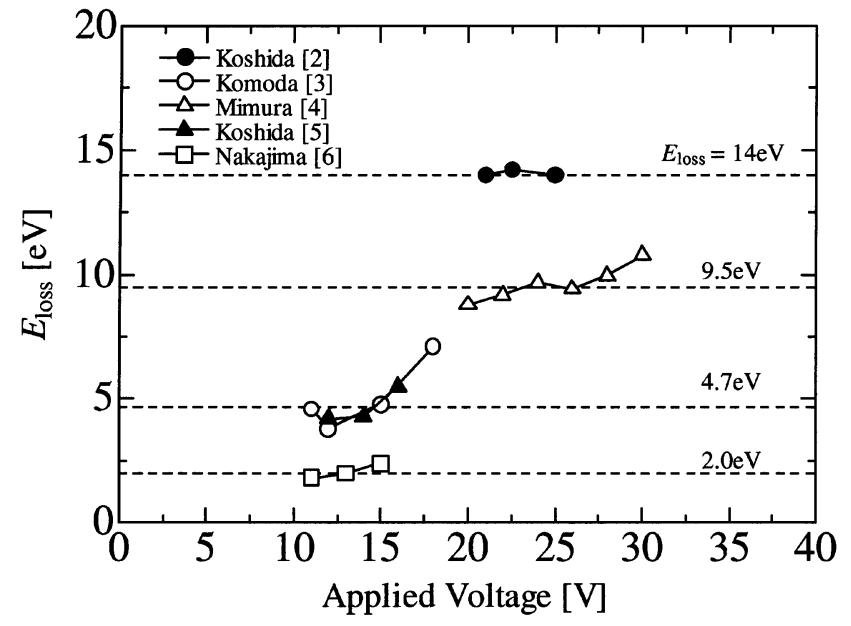

Fig. 4. The $E_{\text {loss }}$, defined in Fig. 2, calculated from the data shown in Fig. 3, being nearly independent of the applied voltage, $V_{\mathrm{ps}}$.

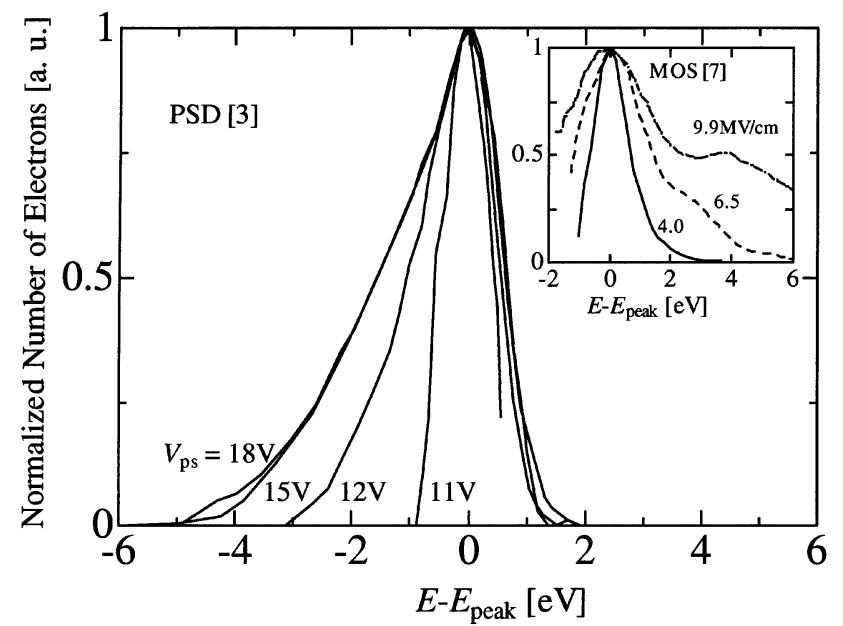

Fig. 5. Impact of increasing applied voltage $V_{\mathrm{ps}}$ on the emission energy distribution observed for PS diode. The horizontal axis is the energy measured from the peak energy, and the vertical axis is normalized so that the number of electrons at the peak equals unity. The inset is the same plot for a MOS emitter.

from that observed in the conventional metal-oxide-semiconductor (MOS) electron emitters [7], where $V_{\mathrm{ps}}$ has significant influence on the distribution shape at the energies larger than $E_{\text {peak }}$, as shown in the inset.

The clarifications shown above facilitate assessments of possible models. Mimura et al. proposed that nc-Si dots on the emitting surface act as nano-scale field emitter tips [4], which is analogous to the Spindt cathode array [8]. This model, however, fails to explain two observations of the emission phenomena. Firstly, the model cannot explain the constant nonzero $E_{\text {loss }}$ values shown in Fig. 4. It is well known that the Fowler--Nordheim $(\mathrm{FN})$ tunneling is the dominant mechanism in the conventional field emission [9]. As there is no energy loss during FN tunneling through vacuum, majority of emitted electrons have the energy near the bottom of the conduction band at the emitting nc-Si. The $E_{\text {loss }}$ values should then be nearly zero when there is no voltage drop across the PS region. When the voltage drop is not negligible, it should be strongly dependent on $V_{\mathrm{ps}}$ because diode current significantly changes with $V_{\mathrm{ps}}$ [1], resulting in strongly voltage-dependent $E_{\text {loss. }}$. Secondly, the field emission model cannot explain the broad energy distribution of PS 


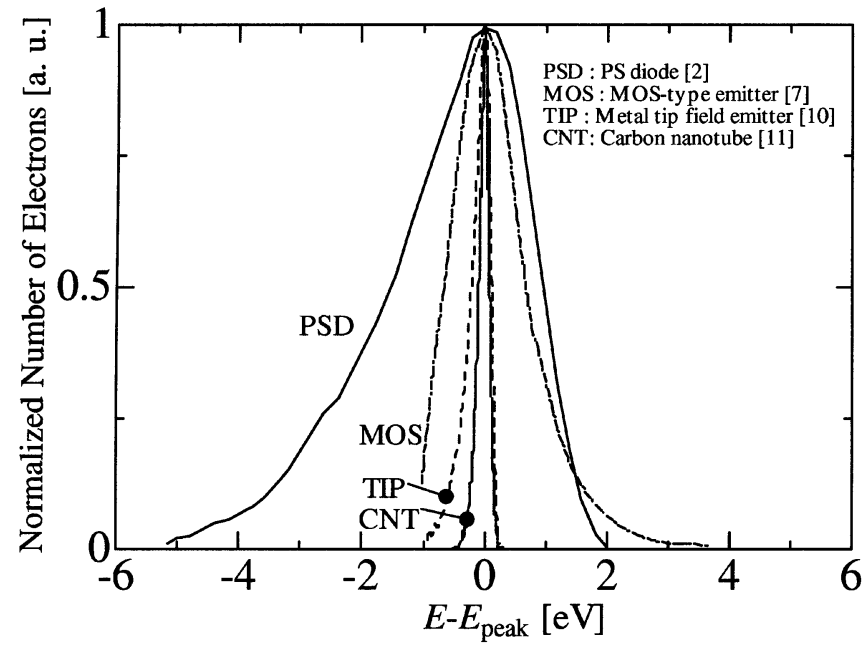

Fig. 6. Comparison of energy distribution shapes observed for various electron emitters.

diode. Fig. 6 compares the energy distribution shape of different electron emitters. The energy dispersion of the PS diode (PSD) is much larger than those of the conventional field emitters, that is, the carbon nanotube (CNT) and the metal tip emitter (TIP) [10], [11]. Thus, the conventional field emission model cannot explain some of the important features of the electron emission from the PS diode.

One may notice that the emission energy distribution of the MOS emitter shown in Fig. 6 has similar energy dispersion as that of the PS diode. Indeed, in oxidized PS diode, large number of nc-Si dots are fully oxidized in the vicinity of the emitting surface [12], so that the PS region may be effectively modeled as bulk $\mathrm{Si}$ with $\mathrm{SiO}_{2}$ layer on top of it. However, the energy distribution of PS diode and MOS emitter have different dependence on $V_{\mathrm{ps}}$ as shown in Fig. 5, indicating that the MOS model also fails to explain the electron emission from the PS diode.

Thus, the conventional electron emission models cannot explain the emission from the nc-Si system. This leads us to discuss another model, in which another physics plays an important role.

\section{A Possible Mechanism of SupPRessed Electron ENERGY RELAXATION IN THE nc-Si DOTS}

A recently suggested phenomenological model asserts that the electrons injected into the PS region can travel for a long distance quasiballistically by multiple tunneling, which is called quasiballistic emission model [2]. In this model, the smallness of the nc-Si dots and strong electric field applied to the nc-Si system are considered to play important roles for the quasiballistic transport [2]. This concept is qualitatively supported by experimental results of the time-of-flight measurement [13], [14] and the strong temperature dependence of emitted electron energy distribution [5], [6], [15]. However, microscopic discussion has not been given yet, and therefore, this model cannot be quantitatively assessed at the present. Instead, we calculate electronic and phononic states in the nc-Si system to examine the possibility of the suppression of energy relaxation. These calculations also give a microscopic footing for more quantitative discussion of the quasiballistic emission model.

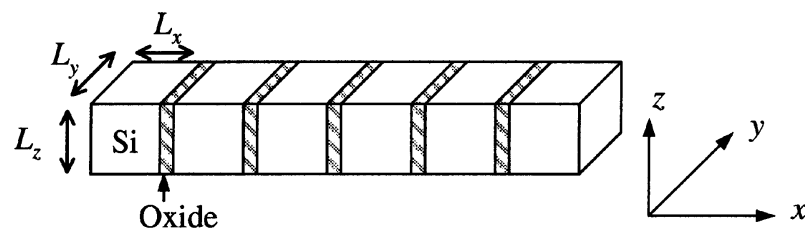

(a)

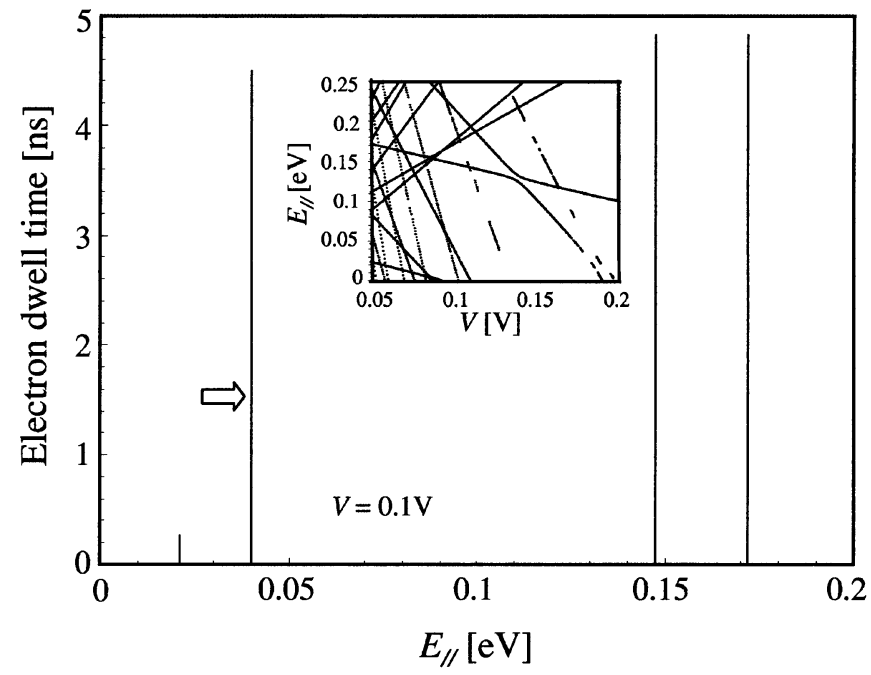

(b)

Fig. 7. (a) The electron states are calculated in a linear chain of quantum dots under an electric field. (b) Electron dwell time as a function of the electron energy in direction to a linear chain of quantum dots $E_{/ /}$. The inset shows the peak energies plotted as a function of voltage applied across each nc-Si dot. The number of quantum dots is 30 .

Firstly, electronic states in the nc-Si system were calculated by solving the Shrödinger equation in a linear chain of nc-Si dots under an electric field. Fig. 7(a) illustrates the model used in our calculation, where $\mathrm{Si}$ dots were modeled as cubes of $L_{x}, L_{y}$, and $L_{z}$. The transverse wave functions are expressed as sinusoidal functions, and the longitudinal wave function is described by the Airy function, asymptotic form of which defines phase shift in this open scattering system. The electron dwell time is the time for which an electron stays among barriers, and given by the differentiation of the phase shift by the electron energy. Fig. 7(b) shows the calculated dwell time as a function of the electron energy in direction to a linear chain of quantum dots $E_{/ /}$, where the total electron energy $E_{\text {total }}$ is given by the sum of $E_{/ /}$and the electron energy perpendicular to a linear chain, that is, $E_{\text {total }}=E_{/ /}+\hbar^{2}\left\{\left(i \pi / L_{y}\right)^{2}+\right.$ $\left.\left(j \pi / L_{z}\right)^{2}\right\} / 2 m^{*},(i, j=1,2, \ldots)$. . Based on structural and optical studies of the PS diodes [12], [16] the size of the silicon dots were assumed to be $4.0 \mathrm{~nm}$, and the oxide thickness and the barrier height were set $1.0 \mathrm{~nm}$ and $1.0 \mathrm{eV}$, respectively. The voltage across each dot was set $0.1 \mathrm{~V}$, which is a typical value under device operation. The calculated dwell time sharply increases up to several ns at certain values of $E_{/ /}$, while it is less than $0.1 \mathrm{ps}$ elsewhere. These peaks represent quasibound states in the nc-Si dots. The peak energies are plotted in the inset as a function of voltage applied across each nc-Si dot. Fig. 8 shows the potential profile and the wave function at a resonance denoted by the 


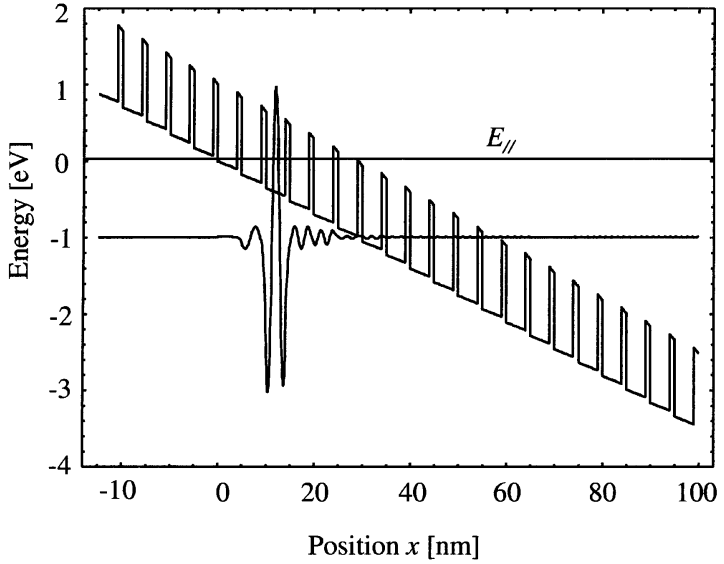

Fig. 8. Potential profile and the wave function at a resonance denoted by the arrow in Fig. 7.

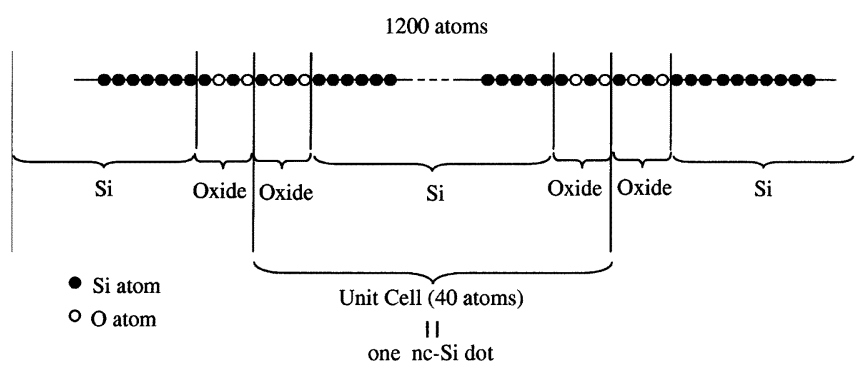

(a)

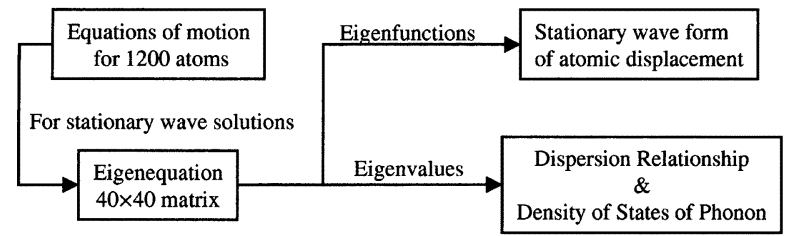

(b)

Fig. 9. (a) Schematic illustration of the simplified model used for phonon calculation. (b) Simulation procedure.

arrow in Fig. 7. Note that the wave function is virtually localized in one quantum dot under the applied voltage, leading to extremely sharp resonance peaks in Fig. 7.

Secondly, the phonon normal modes in the nc-Si system were calculated using the linear chain model, that is, only one-dimensional longitudinal displacements were taken into account. As each nc-Si in the PS diode can be regarded as Si dot surrounded by thin oxide layer [2], we considered a superlattice of Si layer and oxide layer as shown in Fig. 9(a). The mass of Si and O atoms were set based on their atomic weights, that is, 1.0 and $8 / 14$, respectively. The spring constants between $\mathrm{Si}-\mathrm{Si}$ and $\mathrm{Si}-\mathrm{O}$ bonds were assumed based on the Young's modulus of bulk $\mathrm{Si}$ and $\mathrm{SiO}_{2}$, that is, 1.0 and 0.4 , respectively. For simplicity, the distance between two atoms is set unity. We considered a chain of 1200 atoms satisfying the periodic boundary condition. One unit cell contains 40 atoms, and each remote end of the unit cell is defined as oxide layer. The oxide layer was assumed to comprise two $\mathrm{Si}$ and two $\mathrm{O}$ atoms alternating with each other, that is, $\mathrm{Si}-\mathrm{O}-\mathrm{Si}-\mathrm{O}$. The calculation procedure is shown in Fig. 9(b). The phononic states can be obtained by solving the equations

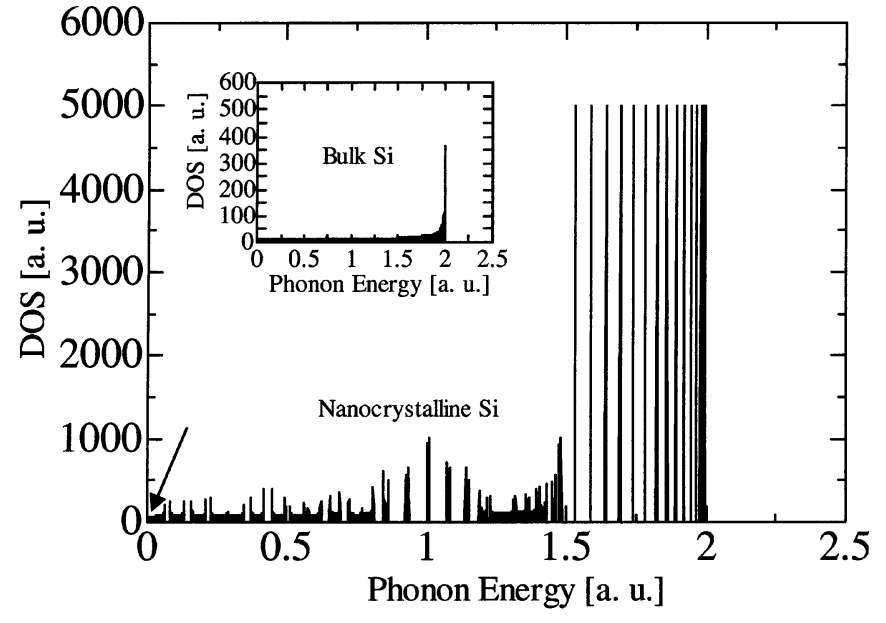

Fig. 10. Density of states of the phonons in a chain of nanocrystalline Si dots plotted as a function of phonon energy. The inset shows the result calculated for bulk Si.

of motion for 1200 atoms. We assumed that only neighboring atoms interact, and that the potential energy is given as the quadratic form of displacement as in harmonic oscillators. The stationary wave solutions are given by solving an eigenequation, dimension of which can be reduced to 40 by taking the translational symmetry into account. Eigenfunctions give the wave forms of atomic displacement, and eigenvalues plotted as a function of wave vector give dispersion relationship and density of states of phonon. Fig. 10 shows the density of states (DOS) as a function of phonon energy, and the inset shows that of bulk Si for comparison. Note that the DOS of the nc-Si system shows energy gaps. Especially, the DOS is fully quantized in the high-energy (frequency) region, showing several sharp peaks. In this region, neighboring atoms displace in opposite directions, and displacements vanish in the oxide layers. This is analogous to the phonon confinement known for superlattice structure of III-V compound semiconductors. In this paper, we refer to such phonon modes as "confined optical phonons", and the other modes as "acoustic phonons". Fig. 11(a) shows displacements of 1200 atoms calculated for an acoustic phonon mode at the arrow in Fig. 10. Note that the displacement wave form is slightly different from the plain wave seen in bulk Si. Fig. 11(b) shows the strain caused by the phonon, which is defined as the differentiation of the displacements by position. The solid line shows the strain in the nc-Si system, and the broken line shows the result calculated for bulk Si. It is important to note that the strain in the nc-Si system has large amplitude in the oxide layers compared to that in $\mathrm{Si}$ regions. As a result, the strain in the $\mathrm{Si}$ regions has smaller amplitude in the nc-Si system than in the bulk $\mathrm{Si}$. In other words, in the nc-Si system, the strain in the $\mathrm{Si}$ regions is weakened due to the mechanically soft oxide layers.

Calculations demonstrated above indicate a possibility of suppression of electron energy relaxation in the nc-Si system. As seen in Fig. 8, the electron wave length varies in space under an electric field. Scattering rate should then decrease unless the transition occurs between resonant states. This energy selection may restrict electron energy relaxation in the nc-Si system. Similarly, Fig. 10 and 11 indicate reduction of the phonon scattering rate for electrons confined in nc-Si dots. 

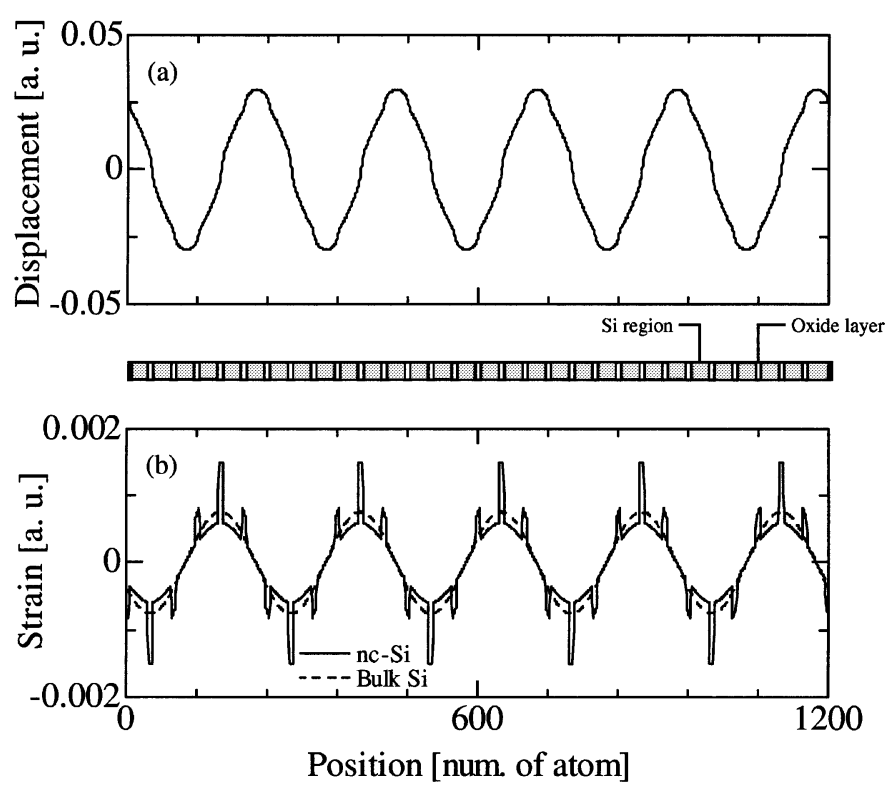

Fig. 11. (a) Displacement of an acoustic phonon normal mode denoted by the arrow in Fig. 10. (b) Strain due to the acoustic phonon displacement plotted for the nc-Si system (solid line) and the bulk Si (broken line).

As the average diameter of nc-Si dots is about $4 \mathrm{~nm}$ [12], [16] the energies of a confined electron are well separated due to quantum effect. For scattering process mediated by high-frequency phonons, the discrete nature of the energy spectrum of electrons and confined optical phonons reduces the number of allowed transitions, during which the energy conservation must hold. This is analogous to the phonon bottleneck phenomenon observed for the III-V semiconductor quantum dot structures [17]. For scattering process via low-frequency phonons, the reduction of strain in Si layer observed in Fig. 11 leads to smaller deformation potential in the nc-Si dots. As the wave function of confined electron has small amplitude in the oxide layers, the concentration of the deformation potential in the oxide layers causes reduction of acoustic phonon scattering rate in the Si dots. Thus, calculations of electronic and phononic states both suggest a possibility of suppression of electron energy loss process mediated by phonons in the nc-Si system.

It should be noted that the nc-Si linear chain model used in our calculation is not too idealistic, because TEM images indeed show that the nc-Si dot size is relatively identical, and that a quasione-dimensional chain of the nc-Si dots is formed as seen in of [12, Fig. 11]. The nc-Si dot linear chain can be observed commonly for high porosity porous silicon grown on a p-type Si substrate [18], followed by natural oxidation. This structure is also obtained by anodizing n-type Si under light illumination [2], [3], [19], resulting in the same structure of high porosity porous layer. This anodization technique is widely used for achieving highly efficient electron emission [1]-[6], and our model is applicable for such quasione-dimensional structures. The imperfect translational symmetry in real structures may smear out the sharp peaks of the optical phonon energy spectrum observed in Fig. 10. However, the optical phonon vibration is still well confined in each Si dot, and it can be approximated as vibration in an isolated Si dot. Therefore, the phonon energy spectrum is still discrete as long as local phonon vibration confined in one $\mathrm{Si}$ dot is concerned, which lead to the reduction of electron-phonon scattering rate. Similarly, the concentration of acoustic phonon strain in oxide layers should still exist even with a lack of the perfect translational symmetry, because this occurs mainly due to the softness of the oxide layers. For these reasons, we believe that our present theoretical model still gives a reasonable description for the real porous silicon despite its simplicity.

We should also discuss the influence of the impact ionization scattering on the high-energy electron emission from the PS diodes. In the bulk Si, the impact ionization scattering becomes comparable to the electron-phonon scattering at energies more than $5 \mathrm{eV}$ [20]. In this case, the impact ionization scattering may also affect the energy loss characteristics of the high-energy electrons. However, the electrons have such a high energy only at the vicinity of the emitting surface. Therefore, we consider that the suppression of the electron-phonon scattering plays the major role in the reduction of electron energy relaxation process.

\section{CONCLUSION}

The electron emission from the nanocrystalline Si dot system, implemented as porous silicon diode, was quantitatively investigated. The relationship between the peak energy measured from the vacuum level, $E_{\text {peak }}$, and the voltage applied to the PS diode, $V_{\mathrm{ps}}$, revealed that the energy difference between the Fermi level at the silicon substrate and the peak energy, $E_{\text {loss }}$, is nearly independent of $V_{\mathrm{ps}}$. The increasing $V_{\mathrm{ps}}$ was found to have significant impact on the shape of the energy distribution at the energies smaller than $E_{\text {peak }}$, while it has minimal impact at larger energies. These clarifications revealed that both the conventional field emission model and the MOS model fail to explain the electron emission from the nc-Si system. This led us to discuss the quasiballistic emission model, which assumes suppression of electron energy relaxation in the nc-Si system. The calculations of electron wave functions and phonon normal modes in a chain of the nc-Si dots indicated a possibility of suppressed electron energy relaxation, which supports quasiballistic emission model. More quantitative discussions and further calculations of the electron-phonon interaction will be given in the following paper.

\section{ACKNOWLEDGMENT}

The authors would like to thank Dr. Z. A. K. Durrani and Mr. M. Khalafalla of Microelectronics Research Centre, University of Cambridge, for their helpful discussions. The authors also have benefited from much useful suggestions and discussions with Dr. T. Shimada of Hitachi Central Research Laboratory, Hitachi Ltd., Dr. M. Ando of Advanced Research Laboratory, Hitachi Ltd., and Prof. S. Oda of Tokyo Institute of Technology.

\section{REFERENCES}

[1] N. Koshida, T. Ozaki, X. Sheng, and H. Koyama, "Cold electron emission from electroluminescent porous silicon diodes," Jpn. J. Appl. Phys., pt. 2, vol. 34, no. 6A, pp. L705-L707, 1995.

[2] N. Koshida, X. Sheng, and T. Komoda, "Quasiballistic electron emission from porous silicon diodes," Appl. Surf. Sci., vol. 146, pp. 371-376, 1999. 
[3] T. Komoda, X. Sheng, and N. Koshida, "Mechanism of efficient and stable surface-emitting cold cathode based on porous polycrystalline silicon films," J. Vac. Sci. Technol. B, vol. 17, no. 3, pp. 1076-1079, 1999.

[4] H. Mimura, K. Miyajima, and K. Yokoo, "Electron emission from porous silicon planar emitters," in Tech. Dig. 15th Int. Vacuum Microelectronics Conf. Lyon, France, 2002. PM18.

[5] N. Koshida, A. Kojima, Y. Nakajima, T. Ichihara, Y. Watabe, and T. Komoda, "Surface-emitting ballistic cold cathodes based on nanocrystalline silicon diodes," presented at the Electro-chemical Society 201st Meeting, vol. 463, Philadelphia, PA, United States, 2002.

[6] Y. Nakajima, A. Kojima, and N. Koshida, "Generation of ballistic electrons in nanocrystalline porous silicon layers and its application to a solid-state planar luminescent device," Appl. Phys. Lett., vol. 81, no. 13, pp. 2472-2474, 2002.

[7] S. D. Brorson, D. J. DiMaria, M. V. Fischetti, F. L. Pesavento, P. M. Solomon, and D. W. Dong, "Direct measurement of the energy distribution of hot electrons in silicon dioxide," J. Appl. Phys., vol. 58, no. 3, pp. 1302-1313, 1985.

[8] C. A. Spindt, C. E. Holland, A. Rosengreen, and I. Brodie, "Field-emitter-array development for high-frequency operation," J. Vac. Sci. Technol. B., vol. 11, no. 2, pp. 468-473, 1993.

[9] D. Nicolaescu, "Technological parameters distribution effects on the current-voltage characteristics of field emitter arrays," J. Vac. Sci. Technol. B., vol. 12, no. 2, pp. 759-763, 1994.

[10] S. T. Purcell and V. T. Binh, "64 meV measured energy dispersion from cold field emission nanotips," Appl. Phys. Lett., vol. 67, no. 3, pp. 436-438, 1995

[11] J. M. Bonard, J. P. Salvetat, T. Stöckli, L. Forró, and A. Châtelain, "Field emission from carbon nanotubes: Perspectives for applications and clues to the emission mechanism," Appl. Phys. A, vol. 69, pp. 245-254, 1999.

[12] A. G. Cullis, L. T. Canham, and P. D. J. Calcott, "The structural and luminescence properties of porous silicon," J. Appl. Phys., vol. 82, no. 3, pp. 909-965, 1997.

[13] O. Klima, P. Hlinomaz, A. Hospodková, J. Oswald, and J. Kočka, "Transport properties of self-supporting porous silicon," J. Noncryst. Sol., vol. 164-166, pp. 961-964, 1993.

[14] A. Kojima and N. Koshida, "Evidence of enlarged drift length in nanocrystalline porous silicon layers by time-of-flight measurements," Jpn. J. Appl. Phys., pt. 1, vol. 40, no. 4B, pp. 2779-2781, 2001.

[15] X. Sheng, A. Kojima, T. Komoda, and N. Koshida, "Efficient and ballistic cold electron emission from porous polycrystalline silicon diodes with a porosity multilayer structure," J. Vac. Sci. Technol. B, vol. 19, no. 1, pp. 64-67, 2001

[16] H. Tanino, A. Kuprin, H. Deai, and N. Koshida, "Raman study of freestanding porous silicon," Phys. Rev. B., vol. 53, no. 4, pp. 1937-1947, 1996.

[17] H. Benisty, "Reduced electron -phonon relaxation rates in quantum-box systems: Theoretical analysis," Phys. Rev. B., vol. 51, no. 19, pp. 13 281-13 293, 1995

[18] R. L. Smith and S. D. collins, "Porous silicon formation mechanisms," J. Appl. Phys., vol. 71, no. 8, pp. R1-R22, 1992.

[19] N. Koshida and H. Koyama, "Efficient visible photoluminescence from porous silicon," Jpn. J. Appl. Phys., vol. 30, no. 7B, pp. L1221-L1223, 1991.

[20] M. V. Fischetti and S. E. Laux, "Understanding hot-electron transport in silicon devices: Is there a shotcut?," J. Appl. Phys., vol. 78, no. 2, pp. 1058-1087, 1995.

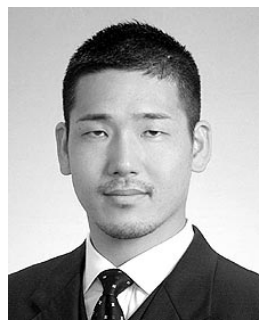

Shigeyasu Uno was born in Kyoto, Japan, on February 10, 1973. He received the B.S. degree in physics from Kwansei Gakuin University, Hyogo, Japan, and the M.S. degree in physics and Ph.D. degree in electronic engineering from Osaka University, Osaka, Japan, in 1996, 1998, and 2002, respectively.

After postdoctoral research at Osaka University, Osaka, Japan, he joined Hitachi Cambridge Laboratory of Hitachi Europe Ltd., Cambridge, U. K. in 2002. His research activity includes the carrier transport and the electron emission of nanostructures.

Dr. Uno received the Edward H. Nicollian Best Student Paper Award from the IEEE Semiconductor Interface Specialists Conference (SISC) and the Young Researcher Award from the International Conference on Solid State Devices and Materials (SSDM), both for his work on tunnel current through the gate oxide.

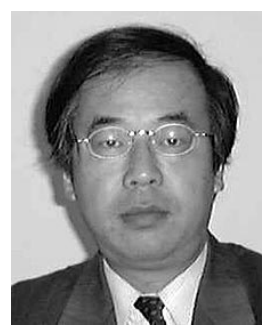

Kazuo Nakazato was born in Japan in $1952 . \mathrm{He}$ received the B.S., M.S., and Ph.D. degrees in physics from the University of Tokyo in 1975, 1977, and 1980.

In 1981 he joined the Central Research Laboratory, Hitachi Ltd., Tokyo, working on high-speed silicon self-aligned bipolar devices, which were adopted in main frame computer Hitachi M-880/420. In 1989, he moved to Hitachi Cambridge Laboratory, Hitachi Europe Ltd., Cambridge, England, as senior researcher and laboratory manager, working on experimental and theoretical study of quantum electron transport in nanometer structures. Since 1997, he has been a chief researcher of Hitachi Cambridge Laboratory. His main research interests are in single-electron and small electron-number memory and logic devices.

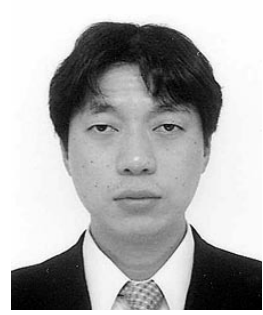

Shinya Yamaguchi was born in Niigata, Japan, on November 30, 1963. He received the B.S. degree in physics from the Sophia University, Tokyo, Japan, in 1987, and the M.S. and Ph.D. degrees in physics from the University of Tokyo, Tokyo, Japan, in 1994 and 1997, respectively. In 1997, he joined Central Research Lab., Hitachi Corporation, Tokyo, Japan, where he worked on epitaxial growth of $\mathrm{Si} / \mathrm{SiGe}$ heterostructure and its application for High-electron Mobility Transistor. He is currently involved in crystallization and technology integration for low-temperature poly-Si Thin-film-transistors. He is the author or co-author of about 50 journal and conference papers and holds 12 US patents.

Mr. Yamaguchi is a Member of the Japan Society of Applied Physics.

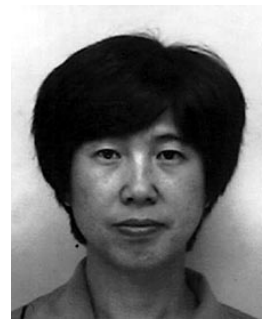

Akira Kojima received the Ph.D. from Tokyo University of Agriculture and Technology, Japan, in 2003.

Since 2003, he has worked as Technical Manager in Quantum $14 \mathrm{KK}$, Tokyo, Japan. He is currently involved in research on nanocrystalline silicon materials and devices.

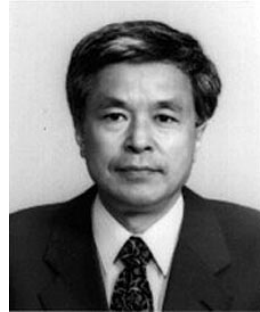

Nobuyoshi Koshida was born in Hokkaido, Japan, in 1943. He received the B.S. and M.S., and Ph.D. degrees in electronic engineering from Tohoku University, Sendai, Japan, in 1966, 1968, and 1973, respectively.

After working as a graduate researcher in Tohoku University and a researcher in the Central Laboratories of Nissan Motor Co. Ltd., Yokosuka, Japan, he moved to Tokyo University of Agriculture and Technology as Associate Professor in 1981. Since 1988, he has been working as Professor of Electronic Device Engineering. Related professional careers are a visiting researcher at MIT, USA, and Cambridge University, UK, in 1992-1993 and a visiting professor at J. Fourier University Grenoble, France, in 1996. His research activity concerns photonic and electronic functions of quantum-sized nanosilicon, its device physics and nanofabrication technology.

Dr. Koshida is a Council Committee Member of Japan Society of Applied Physics, Japan Local Section Chairman of Electrochemical Society. He is also a member of Material Research Society and American Physical Society. 


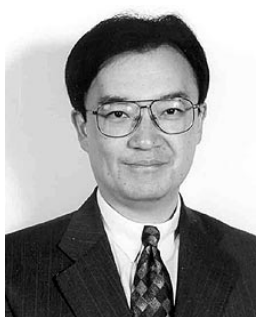

Hiroshi Mizuta was born in Kochi, Japan, in 1961.

He received the B.S. and M.S. degrees in physics and the $\mathrm{Ph} . \mathrm{D}$. degree in electrical engineering from Osaka University, Osaka, Japan, in 1983, 1985 and 1993, respectively.

He joined the Central Research Laboratory, Hitachi, Ltd., Tokyo, Japan in 1985, and has been engaged in research on high-speed compound semiconductor devices as well as study of resonant tunnelling devices. From 1989 to 1991, he has worked on nonequilibrium quantum transport simulation, and also since 1997, he has been working on single-electron devices, advanced silicon memory devices, and nanoelectronics as a laboratory manager and senior researcher at the Hitachi Cambridge Laboratory, Hitachi Europe Ltd., Cambridge, England. He is author of The Physics and Applications of Resonant Tunnelling Diodes, (Cambridge, MA: Cambridge University Press, 1995).

Dr. Mizuta is a Member of the Physical Society of Japan, the Japan Society of Applied Physics, the Institute of Physics and the Electron Devices Society of the IEEE. 